\title{
Vibration characteristics of a rotor-bearing system caused due to coupling misalignment - a review
}

\author{
Ashutosh Kumar', Prabhakar Sathujoda ${ }^{2}$, Vinayak Ranjan ${ }^{3}$ \\ Department of Mechanical and Aerospace Engineering, Bennett University, Greater Noida, India \\ ${ }^{1}$ Corresponding author \\ E-mail: ${ }^{1} a k 7688 @ b e n n e t t . e d u . i n,{ }^{2}$ prabhakar.sathujoda@bennett.edu.in, ${ }^{3}$ vinayak.ranjan@bennett.edu.in
}

Received 30 August 2021; received in revised form 19 September 2021; accepted 28 September 2021 DOI https://doi.org/10.21595/vp.2021.22195

Check for updates

Copyright $(\subset) 2021$ Ashutosh Kumar, et al. This is an open access article distributed under the Creative Commons Attribution License, which permits unrestricted use, distribution, and reproduction in any medium, provided the original work is properly cited.

\begin{abstract}
In this paper, the authors presented various insights into theoretical and experimental analysis carried out in past, to understand the vibration characteristics of a misaligned rotor-bearing system. The literature presented by various researchers was reviewed methodically. Firstly, the literature review based on theoretical vibration analysis of misaligned rotor system with emphasis on finite element method has been presented. Secondly, various vibration-based analysis methods and the description of experimental measurement techniques have been discussed in detail. Apart from the above, distinct tools used for the detection of fault analysis of the rotor system is also reviewed systematically, which may be useful for preventive maintenance of the rotating machinery used in several industries.
\end{abstract}

Keywords: flexible-coupling, misalignment, rotor dynamics, vibration analysis, finite element method (FEM), fault analysis.

\section{Introduction}

Coupling misalignment is the most common fault that occurs in a rotor-bearing system. A typical rotor-bearing system is shown in Fig. 1, which consists of a shaft and disk mounted on the bearings, coupling, drive motor. Perfect alignment of the driving and driven shaft is rarely achieved in practical applications. Even if a perfect alignment is achieved initially, maintaining it for a longer period is not achievable. Various reasons are foundation setting, thermal expansion/contraction of foundation, unbalance, wear and tear, temperature changes arising due to friction or due to process. All couplings subjected to misalignment produces reaction forces and torque on the coupled machine. It is not the misalignment that causes the vibration, but the reaction forces that a misaligned coupling exerts on the machine that provokes the machine to vibrate. Mechanical alignment couplings specifically gear or grid coupling, impose a bending moment on the machines, which is the function of the transmitted torque. Flexible element coupling such as pin and bush coupling enforce a bending moment on the machines, which is proportional to the misalignment but is sovereign of the transmitted torque.

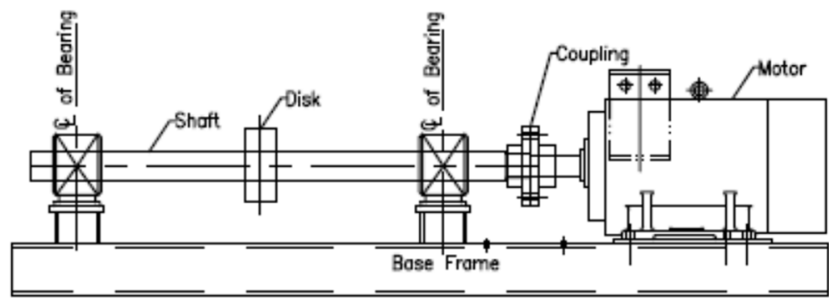

Fig. 1. A typical rotor-bearing system showing shaft, disc, support bearings, coupling and drive motor

A lot of research has been performed to interpret the vibration response behaviour of a rotor-bearing system. It is evident from the available literature that the first harmonic response is due to the presence of unbalance in the rotor system. The unbalanced force is directly proportional to the square of the rotating speed. Many researchers perceived that the second harmonic response 
gives a strong signal for the misalignment of the shaft. However, less literature shows that some other rotor system faults such as rotor friction or rotor cracks also produces the second harmonic response. Few researchers found no indication of the presence of a second harmonic response in their research and designated that no universal rule for shaft misalignment identification exists. The vibration response characteristics have not been understood comprehensively, especially the relation between forces or torques and displacement, accelerations or moments. This review paper gives diverse insights into analyses carried out by researchers in past. The various works of literature have been systematically reviewed to figure out the effect of coupling misalignment on the rotor-bearing system. The objective of this paper is to study numerous analysis models used for the dynamic analysis of a rotor-bearing system with coupling misalignment. In Section 2, the rotor-bearing system model with coupling misalignment based on finite element method (FEM) and in Section 3, Diagnostic analysis and experimental investigations are presented. Finally, the conclusion is drawn in Section 4.

\section{Rotor bearing system model based on FEM}

Gibbons [1] presented a mechanical model to calculate the forces and moments originating from misalignment and analyzed the various types of coupling. Mancuso et. al. [2] derived that a gear or grid coupling, impose a bending moment on the machines, which is a function of the transmitted torque. In the case of pin and bush coupling, the bending moment is proportional to the misalignment but independent of the transmitted torque. The bending moment varies with the bending stiffness of the coupling, which depends on the type of flexible element used in the coupling. Later these force equations based on the analysis proposed by him was adopted by Sekhar and Prabhu [3]. Sudhakar and Sekhar [4] identified the forces and moments developed by misaligned gear coupling and summarized the different studies on coupling misalignment modelling, effects and identification. Bloch and Geitner [5] have provided a critical assessment for couplings concerning the various types of stresses and the comparative values of these forces were declared. Nelson and McVaugh [6] presented a procedure for dynamic modelling of a rotor-bearing system consisting of a rigid disk, rotor element and discrete bearing. Saavedra and Ramirez [7, 8] proposed a new coupling stiffness matrix considering five degrees of freedom per node, three translational displacements each in $x, y$, and $z$-direction and two rotational displacements in the direction perpendicular to the axis of rotation. Extending the work of [6] and discarding the effect of the transverse mass moment of inertia and the gyroscopic effect on the shaft element, the equation of motion for shaft, disc and bearing elements were deduced. A coupling stiffness matrix is defined, to include the coupling element within the finite element analysis of a misaligned rotor system and to determine the vibratory response of misaligned shaft rotors connected by a love joy coupling and a three-pin coupling. Saavedra and Ramirez presumed that the stiffness co-efficient for a specific type of flexible coupling depends on the transmitted torque, the rotation speed, the rotation angle, the misalignment displacement and the misalignment angle. After the qualitative and numerical analysis, it was reported that vibration generated by shaft misalignment is caused by a variation in coupling stiffness and the forcing frequencies generated by shaft misalignment and third-order harmonic frequency is dominant in the vibration spectrum.

Patel and Darpe $[9,10]$ outlines a method of determination of the misalignment excitation by investigating a coupled rotor-bearing system with pin-bush flexible coupling. Misalignment forces and moments were obtained experimentally using a six-axis load-cell and implemented in a finite element model. All three moments and three forces were measured and used to formulate a more refined and realistic coupling stiffness matrix with all six degrees of freedom per node. The researchers reported that the majority of the misalignment forces induce higher harmonics and out of them, the $3 \mathrm{X}$ frequency component is more prominent. Also, the forces vary continuously during rotation with a large mean force acting due to the misalignment. The presence of strong higher harmonic frequency components are strong contenders for unique identification 
of rotor misalignment and can distinguish misalignment from rotor crack.

During the analysis of a stepped beam, Balasubramanian and Subramanian [11] developed a beam element having deflection, slope, bending moment and shear force as degrees of freedom at each node. The stiffness and mass matrix for stepped beam analysis were calculated. To study the vibration response of misaligned coupling, Sekhar and Prabhu [3] extended the analysis of [11] to two planes, considering beam elements having eight degrees of freedom at each node. A higherorder finite element was thus presented. The beam deflection $q(x)$ for an element of length 1 is expressed as the seventh degree of a polynomial in $x$. Kramer [12] defined two models for coupling, the first model considering the flexibility of mechanical coupling as rigid in a radial direction and the second model considering the rotational stiffness and damping of the coupling. Nelson and Crandall [13] also envisaged the coupling with two models, the first one considering the couplings as an elastic component with isotropic translational and rotational stiffness and the second model incorporating the internal damping and the inertia effects, in addition to coupling stiffness. The four different models as shown in Fig. 2 have been experimentally investigated by Tadeo et al. [14] for a flexible disc Vulkan Tormin L-3R coupling, which allows angular misalignment relatively less than parallel misalignment. Tadeo et al. observed that a second model by Nelson and Crandall, best describes the dynamics of the coupling because it includes rotational stiffness, damping, mass and inertia. However, the second model of Kramer, which includes rotational stiffness and damping only, showed that these coupling parameters are most important for controlling the dynamic response. Tadeo and Cavalca [15] made a comparison of flexible coupling models for getting its response using different models techniques found in the literature of $[12]$ and $[6,13]$. The stiffness and damping parameters of the couplings and the bearings were estimated using the Parameter Estimation method based on the non-linear damped least square method as explained by Arruda and Duarte [16]. The magnitude of frequency response functions was compared with the corresponding magnitude of the Frequency response function of the experimental setup.

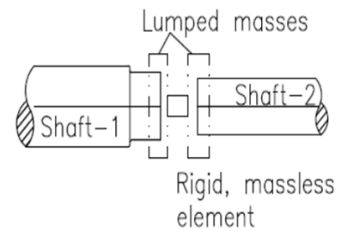

a)

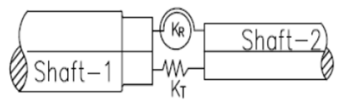

c)

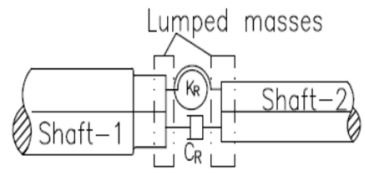

b)

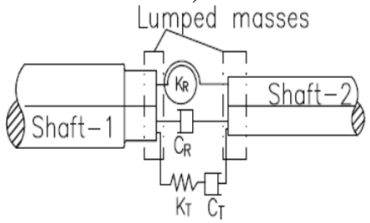

d)

Fig. 2. Flexible coupling models: a) Kramers's first model; b) Kramer's second model; c) Nelson and Crandall's first model; and d) Nelson and Crandall's second model

Using FEM and considering six degrees of freedom, Wang and Gong [17] modelled two coupled rotor systems with three disks, each rotor supported on two bearings. Using nodal force and moment vectors, misalignment effects were considered at the coupling location. The researchers used Newmark- $\beta$ method to solve the non-linear equations, and observed that $2 \mathrm{X}$ will appear in parallel misalignment force spectrum and $2 \mathrm{X}, 4 \mathrm{X}, 6 \mathrm{X}$ will appear in angular misalignment moment simulation. In the case of parallel misalignment, the orbit exhibited an inverted triangle and in angular misalignment simulation, the orbit looked like an eight shape.

The response of a shaft coupler system consisting of flexible shafts, unbalance disc, elastic support and shaft coupler with parallel misalignment were derived using a transfer matrix by Tsai 
and Huang [18]. The result showed that the shaft misalignment prevailed the response in most of the rotation speed, but the unbalance could become momentous at high speed. An illustrative shaft misalignment model incorporating both parallel and angular misalignment with mass unbalance and a flexible coupling having axial, torsional and axial flexibility was presented by Redmond [19], which was able to simulate non-linear bearing stiffness and coupling angular stiffness anisotropy. Lal and Tiwari [20-23] presented a series of literature and developed an identification algorithm, based on the least-square fit technique in the frequency domain, to evaluate parameters of speed-dependent multiple faults in a turbine generator system model having flexible coupling. The authors claimed that the proposed algorithm could be used for simultaneous estimation of bearing dynamic parameters, residual unbalance, and the misalignment forces and moments from the forced response of the turbo-generator system. Recently Kannababu et al. [24] proposed a method using additive coupling stiffness (ACS) for representation and identification of parallel and angular misalignment.

Al-Hussain [25] further proposed a model about angular misalignment affecting the flexible coupling joint connecting two Jeffcott rotors, each mounted on two sets of hydrodynamic bearing that displays asymmetric principal stiffness and cross-coupling terms. The dimensionless stability criteria of the non-linear system of differential equations are obtained using Liapunov's direct method through partial differentiation of Hamiltonians obtained from kinetic and potential energies. Vibration analysis of the rotor-bearing system of a misaligned shaft was studied by Rao and Sekhar [26]. By using the Lagrange method and considering 6 degree-of-freedom (DOF) model, Xia et al. [27] calculated the dynamic reaction force varying with rotational angle by analyzing the deformation of a hexangular flexible coupling connecting two rigid rotors, which have an angular and parallel misalignment. To solve the differential equations and calculate the vibration response of the rotors, the high order Runge-Kutta algorithm is applied.

A dynamic model is derived by Lee and Lee [28] for a misaligned rotor-ball bearing system driven through a flexible coupling by treating the reaction loads and deformations at the bearing and coupling elements as the misalignment effect. Jafari and Jamshidi [29] examined the nonlinear vibration characteristics of a rotor with an asymmetric shaft (rectangular), connected to a motor through a flexible coupling. Xu and Marangoni [30,31] presented a theoretical model of a motor with a versatile flexible coupling rotor for describing the mechanical vibration resulting from angular misalignment. The researchers assumed that the flexible coupling behaved as a universal joint and concluded that the misalignment is often characterized by a $2 \mathrm{X}$ component. However, the misalignment effect might not articulate always because the forcing frequency of misalignment ( $2 \times$ shaft running speed) is not close enough to at least one of the system natural frequencies to excite the system substantially. In some cases, the misalignment response is hidden and doesn't show up within the vibration spectrum.

Mitchell and Dewell [32] determined that the expected vibration frequencies for a misaligned metallic-disc flexible coupling having four bolts were four times (4X) the shaft running speed. Upper sideband frequency is going to be the number of bolts plus shaft's speed i.e. five times (5X) of shaft's speed. Lower sideband fundamental frequency is going to be the number of bolts minus shaft's speed i.e. three times (3X) of the shaft's speed. Tuckmantel et al. [33] investigated the steady-state response of a rotor-journal bearing-flexible disc coupling system by introducing angular misalignment to the system. Its effect on the coupling reaction forces and moments exerted in a coupled shaft is simulated through the model proposed by Sekhar and Prabhu [3], considering only the bending flexibility and disregarding torque. Chen et al. [34] carried out a comprehensive study on the rotor system misalignment, through a conjunction of Workbench and experimental verification using spindle reliability test bench, and axial stiffness and radial stiffness are solved and therefore the formula for extra loads caused due to misalignment is derived. Jalan and Mohanty [35] presented a model-based technique for fault diagnosis of a rotor-bearing system under steady-state condition using the residual generation technique. 


\section{Diagnostic analysis and experimental investigation}

This section provides a summary of varied techniques used for the diagnosis and monitoring of misalignment in a rotor-bearing system. The different techniques used by researchers are vibration monitoring and analysis, noise monitoring, motor current signature analysis (MCSA), artificial neural networks, thermography etc.

Prabhakar et al. [36] studied the transient response of a misaligned rotor-coupling-bearing system using the finite element method. From the vibration analysis, the subcritical speed at one-half, one-third and one-fourth were found when the misaligned rotor-coupling-bearing system passes through its critical speed. The continuous wavelet transform (CWT) has been used to extract the silent features from the time response of the rotor system by Prabhakar [37]. Ganeriwala et al. [38, 39] conducted a series of tests on a Machinery Fault Simulator (MFS) to determine a unique vibration signature of a shaft with coupling misalignment. The researchers concluded that a stiffer coupling always results in a higher vibration level and the coupling geometry plays a very important role. The rigid coupling shows moderate peaks at $2 \mathrm{X}, 4 \mathrm{X}$ and $6 \mathrm{X}$ and a moderate one is at $5 \mathrm{X}$. The presence of $2 \mathrm{X}$ and its harmonics are easier to identify by the geometry of the coupling, but the reason for $5 \mathrm{X}$ remains deceptive.

The systematic and detailed experimentations were performed by Azeem et al. [40] on Spectra Quest's Machinery Fault Simulator for two common faults of the rotating machinery i.e. misalignment and crack. It was reported that the misaligned shaft shows higher vibration amplitude at $2 \mathrm{X}$ shaft running speed. The effect of coupling misalignment on the dynamic characteristics of an unbalance multi-disk rotor supported by oil-lubricated journal bearings are investigated experimentally and theoretically by Wan et al. [41]. The investigators reported that misalignment can cause multiple frequency vibrations, including $2 \mathrm{X}, 3 \mathrm{X}, 4 \mathrm{X}, 5 \mathrm{X}$ and other multiple frequency components of vibration, among which the $2 \mathrm{X}$ is dominant. Tuckmantel and Cavalca [42] carried out a comparison between two approaches for modelling the forces and moments originated by a flexible disc coupling with angular misalignment. The first approach is based on linear bending flexure, as proposed by Sekhar and Prabhu [3], of the disk packs, and assuming that the misalignment effects are the summation of the first four harmonic components. And the second approach is a structural analysis of the coupling through the finite element method Abaqus. The cyclic nature of coupling efforts is captured by the application of consecutive shaft spin angles.

Pennachi and Vania [43] proposed two diagnostic techniques, the orbit shape analysis and the model-based identification in the frequency domain, to identify the faults in rotating machinery and validated through an experimental case study concerning a gas turbine generator unit. Lei et al. [44] provided a comprehensive reference for the researchers concerning Empirical Mode Decomposition (EMD), as a time-frequency analysis technique, which can process nonlinear and non-stationary signals. Lei et al. [45] presented, a new method named adaptive ensemble empirical mode decomposition, in which, the sifting number is adaptively selected, and therefore the amplitude of the added noise changes with the signal frequency component during the decomposition process. Srinivas et al. $[46,47]$ studied the static and time-dependent coupling misalignment forces in flexibly coupled Jeffcott rotor systems with angular misalignment and integrated with active magnetic bearing (AMB). Natraj and Baskaran [48] proposed a method to analyze the vibration behaviour, using Bartlett Power Spectral Density (BPSD), of a rotor-bearing system having coupling misalignment and looseness of bearing faults under the dynamic state. Verrucchi et al. [49] experimented to study the effect of the misalignment in induction motor coupled with flexible coupling employing the Motor Current Signature Analysis (MCSA) and the Load torque Signature Analysis ((LTSA). Hariharan and Srinivasan [50] performed an experimental study on a rotor dynamic test bench to predict the vibration spectrum for shaft misalignment using three-pin type flexible coupling. The experimental and numerical (ANSYS) results were obtained and compared. The authors concluded that misalignment can be characterized primarily by $2 \mathrm{X}$ (two times) shaft running speed. Lu et al. [51] applied the proper 
orthogonal decomposition (POD) method for dimensions reduction of dual rotor-bearing system model with coupling misalignment and compared the dynamical behaviour of the reduced-order model with finite element model and revealed that the POD method is having higher computational efficiency and accuracy. Nicolae et al. [52] employed the optimal auxiliary functions method (OAFM) to investigate the dynamic behaviour generated by angular misalignment of two rotors connected through flexible coupling. Mogal and Lalwani [53] proposed the order analysis technique of vibration analysis for fault diagnosis of unbalance and misalignment of the rotor-bearing system. The researchers used the phase and the amplitude obtained from the order analysis to identify fault type and its location effortlessly. Xin Lu et al. [54] investigated the influence of misalignment on the nonlinear dynamics of a two-shaft rotor-bearing-gear coupling system with rub-impact fault using the time-frequency method. The investigators revealed that the misalignment affects the stability of the rotor system harshly at high rotating speed and with increasing misalignment the speed range of the unstable motion also increases.

Experimental and theoretical investigation about reaction moments was carried out by Cura and Mura [55] for the misaligned splined coupling. The use of Hilbert Huang Transform (HHT) to detect the lowest possible misalignment level on a rotor-coupling-bearing system was studied by Sekhar and Chandra [56]. The researchers concluded that HHT is more efficient than other conventional techniques like Fourier spectrum analysis, Continuous Wavelet Transform (CWT), even for a very small amount of angular misalignment. A combined approach of discrete wavelet transform (DWT) and fuzzy logic was introduced by Umbrajkaar and Krishnamoorthy [57] to anticipate the degree of misalignment (DoM). The DB2 mother wavelet was employed for feature extraction. The researchers adduced that the prediction accuracy of DoM gets enhanced by the incorporation of a fuzzy inference system and attained less than one percent error in the output result of vibration signal analysis. Zhou et al. [58] proposed a statistical fuzzy vector chain code (SFVCC) for extracting the shape feature vectors of the shaft orbit. By utilizing these shape vectors as input of support vector machine (SVM), shaft orbit of different rotating types of machinery were identified. Verma, Sarangi and Kolekar [59] conducted an experimental investigation to detect the misalignment by using diagnostic tools as Stator current signature. Chacon et al. [60] conducted an experimental investigation using Acoustic Emission (AE) technique, to detect shaft angular misalignment. Reddy and Sekhar [61] presented an alternative method to detect misalignment using torque measurement and observed that angular misalignment torque signature and parallel misalignment torque signatures are having different spectral characteristics. Another misalignment detection tool was introduced by Fatima et al. [62, 63], by measuring the rate of temperature rise of shaft coupling through thermal imaging. For detection of wind turbine shaft misalignment, a temperature measurement technique, using an infra-red thermometer was suggested by Tonks and Wang [64]. The model was investigated and validated through a real-time shaft alignment test. The rate of change in temperature was found to be a factor of misalignment. M. Attia Hili et al. [65, 66] studied and developed a model, to quantify the bearing dynamic behaviour and loads that affect bearing. Also, vibration response was characterized to identify the type of misalignment. A model for study and diagnosis of angular misalignment in induction motor-load system was conferred by Bossio et al. [67], and the effects of angular misalignment on motor power, motor torque and therefore the current were simulated. The researchers informed that detected motor variables viz. load, current and instantaneous power can detect the angular misalignment. Additionally, the infra-red thermography was conducted, to evaluate the grimness of the angular misalignment on the flexible coupling.

\section{Conclusions}

The authors have attempted to analyze and classify the kinds of literature mainly of the last few years according to various methodology/modelling methods used by the researchers. From the above literature survey, the authors realized that a lot of research has been carried out to 
interpret the vibration behaviour of misaligned rotor system, however, the authors believe that not enough works are reported when the rotor system is accelerating or decelerating during start-up or shut-down. As many researchers perceived that second harmonic response gives a strong signal for the misalignment of the shaft, however, literature also shows that other types of rotor system malfunction can also produce second harmonic response such as rotor friction or rotor cracks. Few researchers indicated that no universal rule for the identification of shaft misalignment in a vibration spectrum exists and it essentially depends on the type of coupling used, and the System's FRF's. Therefore, only one type of character signal such as vibration signal is not substantial to judge the misalignment, as it is confounding. The current literature makes clear that a comprehensive analysis of the dynamic impact of a flexible coupling misalignment on a real shaft-line with several bearings is lacking, and it could be investigated. In addition to MCSA, LTSA, Thermal imaging, and other characteristic signals, additional characterization signals to be explored for real-time diagnosis of misalignment of the rotor-bearing system.

\section{References}

[1] C. B. Gibbons, "Coupling Misalignment Forces," Texas A\&M University. Gas Turbine Laboratories, pp. 111-116, 1976, https://doi.org/10.21423/r10t1x

[2] J. R. Mancuso, C. B. Gibbons, and R. E. Munyon, "The application of flexible couplings for turbomachinery," Texas A\&M University. Turbomachinery Laboratories, pp. 141-164, 1989, https://doi.org/10.21423/r14m3v

[3] A. S. Sekhar and B. S. Prabhu, "Effects of coupling misalignment on vibrations of rotating machinery," Journal of Sound and Vibration, Vol. 185, No. 4, pp. 655-671, Aug. 1995, https://doi.org/10.1006/jsvi.1995.0407

[4] G. N. D. S. Sudhakar and A. S. Sekhar, "Coupling misalignment in rotating machines: modelling, effects and monitoring," Noise and Vibration Worldwide, Vol. 40, No. 1, pp. 17-39, Jan. 2009, https://doi.org/10.1260/0957-4565.40.1.17

[5] H. P. Bloch and F. K. Geitner, An Introduction to Machinery Reliability Assessment. Van Nostrand Reinhold, 1990.

[6] H. D. Nelson and J. M. Mcvaugh, "The dynamics of rotor-bearing systems using finite elements," Journal of Engineering for Industry, Vol. 98, No. 2, pp. 593-600, May 1976, https://doi.org/10.1115/1.3438942

[7] P. N. Saavedra and D. E. Ramírez, "Vibration analysis of rotors for the identification of shaft misalignment Part 1: Theoretical analysis," Proceedings of the Institution of Mechanical Engineers, Part C: Journal of Mechanical Engineering Science, Vol. 218, No. 9, pp. 971-985, Sep. 2004, https://doi.org/10.1243/0954406041991297

[8] P. N. Saavedra and D. E. Ramírez, "Vibration analysis of rotors for the identification of shaft misalignment Part 2: Experimental validation," Proceedings of the Institution of Mechanical Engineers, Part C: Journal of Mechanical Engineering Science, Vol. 218, No. 9, pp. 987-999, Sep. 2004, https://doi.org/10.1243/0954406041991198

[9] T. H. Patel and A. K. Darpe, "Vibration response of misaligned rotors," Journal of Sound and Vibration, Vol. 325, No. 3, pp. 609-628, Aug. 2009, https://doi.org/10.1016/j.jsv.2009.03.024

[10] T. H. Patel and A. K. Darpe, "Experimental investigations on vibration response of misaligned rotors," Mechanical Systems and Signal Processing, Vol. 23, No. 7, pp. 2236-2252, Oct. 2009, https://doi.org/10.1016/j.ymssp.2009.04.004

[11] T. S. Balasubramanian and G. Subramanian, "On the performance of a four-degree-of-freedom per node element for stepped beam analysis and higher frequency estimation," Journal of Sound and Vibration, Vol. 99, No. 4, pp. 563-567, Apr. 1985, https://doi.org/10.1016/0022-460x(85)90541-3

[12] E. Kramer, Dynamics of Rotors and Foundations. New York: Springer-Verlag, 1993, p. 381.

[13] H. D. Nelson and S. H. Crandall, Analytic Prediction of Rotordynamic, Vol. 2. USA: McGraw-Hill Inc., 1992.

[14] A. T. Tadeo, K. L. Cavalca, and M. J. Brennan, "Dynamic characterization of a mechanical coupling for a rotating shaft," Proceedings of the Institution of Mechanical Engineers, Part C: Journal of Mechanical Engineering Science, Vol. 225, No. 3, pp. 604-616, Mar. 2011, https://doi.org/10.1243/09544062jmes2214 
[15] A. T. Tadeo and K. L. Cavalca, "A comparison of flexible coupling models for updating in rotating machinery response," Journal of the Brazilian Society of Mechanical Sciences and Engineering, Vol. 25, No. 3, pp. 235-246, Sep. 2003, https://doi.org/10.1590/s1678-58782003000300004

[16] J. R. F. Arruda and M. A. V. Duarte, "Updating rotor-bearing finite element models using experimental frequency response functions," Journal of the Brazilian Society of Mechanical Sciences and Engineering, Vol. 15, No. 2, pp. 136-149, 1993.

[17] H. Wang and J. Gong, "Dynamic analysis of coupling misalignment and unbalance coupled faults," Journal of Low Frequency Noise, Vibration and Active Control, Vol. 38, No. 2, pp. 363-376, Jun. 2019, https://doi.org/10.1177/1461348418821582

[18] C.-Y. Tsai and S.-C. Huang, "Transfer matrix for rotor coupler with parallel misalignment," Journal of Mechanical Science and Technology, Vol. 23, No. 5, pp. 1383-1395, May 2009, https://doi.org/10.1007/s12206-008-1216-9

[19] I. Redmond, "Study of a misaligned flexibly coupled shaft system having nonlinear bearings and cyclic coupling stiffness-Theoretical model and analysis," Journal of Sound and Vibration, Vol. 329, No. 6, pp. 700-720, Mar. 2010, https://doi.org/10.1016/j.jsv.2009.09.039

[20] M. Lal and R. Tiwari, "Multi-fault identification in simple rotor-bearing-coupling systems based on forced response measurements," Mechanism and Machine Theory, Vol. 51, pp. 87-109, May 2012, https://doi.org/10.1016/j.mechmachtheory.2012.01.001

[21] M. Lal and R. Tiwari, "Quantification of multiple fault parameters in flexible turbo-generator systems with incomplete rundown vibration data," Mechanical Systems and Signal Processing, Vol. 41, No. 1-2, pp. 546-563, Dec. 2013, https://doi.org/10.1016/j.ymssp.2013.06.025

[22] M. Lal and R. Tiwari, "Experimental estimation of misalignment effects in rotor-bearing-coupling systems," in Proceedings of the 9th IFToMM International Conference on Rotor Dynamics, pp. 779789, 2015, https://doi.org/10.1007/978-3-319-06590-8_64

[23] M. Lal and R. Tiwari, "Experimental identification of shaft misalignment in a turbo-generator system," Sādhanā, Vol. 43, No. 5, pp. 1-20, May 2018, https://doi.org/10.1007/s12046-018-0859-1

[24] S. Srinivas R., R. Tiwari, and C. K. Babu, "Modeling, analysis, and identification of parallel and angular misalignments in a coupled rotor-bearing-active magnetic bearing system," Journal of Dynamic Systems, Measurement, and Control, Vol. 143, No. 1, Sep. 2020, https://doi.org/10.1115/1.4048352

[25] K. M. Al-Hussain, "Dynamic stability of two rigid rotors connected by a flexible coupling with angular misalignment," Journal of Sound and Vibration, Vol. 266, No. 2, pp. 217-234, Sep. 2003, https://doi.org/10.1016/s0022-460x(02)01627-9

[26] A. S. Rao and A. S. Sekhar, "Vibration analysis of rotor - coupling - bearing system with misaligned shafts," in ASME 1996 International Gas Turbine and Aeroengine Congress and Exhibition, pp. 1-8, Jun. 1996, https://doi.org/10.1115/96-gt-012

[27] Y. Xia, J. Pang, L. Yang, Q. Zhao, and X. Yang, "Study on vibration response and orbits of misaligned rigid rotors connected by hexangular flexible coupling," Applied Acoustics, Vol. 155, pp. 286-296, Dec. 2019, https://doi.org/10.1016/j.apacoust.2019.05.022

[28] Y.-S. Lee and C.-W. Lee, "Modelling and vibration analysis of misaligned rotor-ball bearing systems," Journal of Sound and Vibration, Vol. 224, No. 1, pp. 17-32, Jul. 1999, https://doi.org/10.1006/jsvi.1997.1301

[29] A. A. Jafari and P. Jamshidi, "Investigating nonlinear vibration behavior of rotors with asymmetry shaft considering misalignment," Journal of Solid Mechanics, Vol. 11, No. 3, pp. 535-549, Sep. 2019, https://doi.org/10.22034/jsm.2019.666851

[30] M. Xu and R. D. Marangoni, "Vibration analysis of a motor-flexible coupling-rotor system subject to misalignment and unbalance, Part I: theoretical model and analysis," Journal of Sound and Vibration, Vol. 176, No. 5, pp. 663-679, Oct. 1994, https://doi.org/10.1006/jsvi.1994.1405

[31] M. Xu and R. D. Marangoni, "Vibration analysis of a motor-flexible coupling-rotor system subject to misalignment and unbalance, Part II: experimental validation," Journal of Sound and Vibration, Vol. 176, No. 5, pp. 681-691, Oct. 1994, https://doi.org/10.1006/jsvi.1994.1406

[32] D. L. Dewell and L. D. Mitchell, "Detection of a misaligned disk coupling using spectrum analysis," Journal of Vibration and Acoustics, Vol. 106, No. 1, pp. 9-16, Jan. 1984, https://doi.org/10.1115/1.3269161

[33] F. W. S. Tuckmantel, C. G. Schoola, and K. L. Cavalca, "Flexible disc coupling model in rotating shafts," Mechanisms and Machine Science, Vol. 61, pp. 502-517, 2019, https://doi.org/10.1007/9783-319-99268-6_35 
[34] F. Chen, F. Bai, C. Chen, S. Wang, and H. Zhang, "Research on double span rotor system driven by motorized spindle with coupling misalignment," Advances in Mechanical Engineering, Vol. 11, No. 4, p. 168781401882100 , Apr. 2019, https://doi.org/10.1177/1687814018821009

[35] A. K. Jalan and A. R. Mohanty, "Model based fault diagnosis of a rotor-bearing system for misalignment and unbalance under steady-state condition," Journal of Sound and Vibration, Vol. 327, No. 3-5, pp. 604-622, Nov. 2009, https://doi.org/10.1016/j.jsv.2009.07.014

[36] S. Prabhakar, A. S. Sekhar, and A. R. Mohanty, "Vibration analysis of a misaligned rotor-couplingbearing system passing through the critical speed," Proceedings of the Institution of Mechanical Engineers, Part C: Journal of Mechanical Engineering Science, Vol. 215, No. 12, pp. 1417-1428, Dec. 2001, https://doi.org/10.1243/0954406011524784

[37] Prabhakar Sathujoda, "Detection of coupling misalignment in a rotor system using wavelet transforms," International Journal of Aerospace and Mechanical Engineering, Vol. 14, No. 4, pp. 175-180, Mar. 2020.

[38] C. Bai, S. Ganeriwala, and N. Sawalhi, "A rational basis for determining vibration signature of shaft/coupling misalignment in rotating machinery," in Rotating Machinery, Vibro-Acoustics and Laser Vibrometry, Volume 7, Vol. 7, pp. 207-217, 2019, https://doi.org/10.1007/978-3-319-74693720

[39] N. Sawalhi, S. Ganeriwala, and M. Tóth, "Parallel misalignment modeling and coupling bending stiffness measurement of a rotor-bearing system," Applied Acoustics, Vol. 144, pp. 124-141, Jan. 2019, https://doi.org/10.1016/j.apacoust.2017.07.022

[40] N. Azeem, X. Yuan, H. Raza, and I. Urooj, "Experimental condition monitoring for the detection of misaligned and cracked shafts by order analysis," Advances in Mechanical Engineering, Vol. 11, No. 5, p. 168781401985130, May 2019, https://doi.org/10.1177/1687814019851307

[41] Z. Wan, J.-P. Jing, G. Meng, Y. Yang, and H.-Y. Bai, "Theoretical and experimental study on the dynamic response of multi-disk rotor system with flexible coupling misalignment," Proceedings of the Institution of Mechanical Engineers, Part C: Journal of Mechanical Engineering Science, Vol. 226, No. 12, pp. 2874-2886, Dec. 2012, https://doi.org/10.1177/0954406211435582

[42] F. W. D. S. Tuckmantel and K. L. Cavalca, "Vibration signatures of a rotor-coupling-bearing system under angular misalignment," Mechanism and Machine Theory, Vol. 133, pp. 559-583, Mar. 2019, https://doi.org/10.1016/j.mechmachtheory.2018.12.014

[43] P. Pennacchi and A. Vania, "Diagnosis and model based identification of a coupling misalignment," Shock and Vibration, Vol. 12, No. 4, pp. 293-308, 2005, https://doi.org/10.1155/2005/607319

[44] Y. Lei, J. Lin, Z. He, and M. J. Zuo, "A review on empirical mode decomposition in fault diagnosis of rotating machinery," Mechanical Systems and Signal Processing, Vol. 35, No. 1-2, pp. 108-126, Feb. 2013, https://doi.org/10.1016/j.ymssp.2012.09.015

[45] Y. Lei, N. Li, J. Lin, and S. Wang, "Fault diagnosis of rotating machinery based on an adaptive ensemble empirical mode decomposition," Sensors, Vol. 13, No. 12, pp. 16950-16964, Dec. 2013, https://doi.org/10.3390/s131216950

[46] R. Siva Srinivas, R. Tiwari, and C. K. Babu, "Interaction between unbalance and misalignment responses in flexibly coupled rotor systems integrated with AMB," in ASME 2017 Gas Turbine India Conference, pp. 1-11, Dec. 2017, https://doi.org/10.1115/gtindia2017-4535

[47] R. Siva Srinivas, R. Tiwari, and C. Kannababu, "Identification of coupling parameters in flexibly coupled Jeffcott rotor systems with angular misalignment and integrated through active magnetic bearing," in Mechanisms and Machine Science, pp. 221-235, 2019, https://doi.org/10.1007/978-3319-99270-9_16

[48] M. Nataraj and G. Baskaran, "Experimental investigation of misalignment and looseness in rotor bearing system using bartlett power spectral density," Journal of Scientific and Industrial Research, Vol. 76, pp. 308-313, May 2017.

[49] C. Verucchi, J. Bossio, G. Bossio, and G. Acosta, "Misalignment detection in induction motors with flexible coupling by means of estimated torque analysis and MCSA," Mechanical Systems and Signal Processing, Vol. 80, pp. 570-581, Dec. 2016, https://doi.org/10.1016/j.ymssp.2016.04.035

[50] V. Hariharan, "Vibration analysis of misaligned shaft - ball bearing system," Indian Journal of Science and Technology, Vol. 2, No. 9, pp. 45-50, Sep. 2009, https://doi.org/10.17485/ijst/2009/v2i9.11

[51] K. Lu et al., "The applications of POD method in dual rotor-bearing systems with coupling misalignment," Mechanical Systems and Signal Processing, Vol. 150, p. 107236, Mar. 2021, https://doi.org/10.1016/j.ymssp.2020.107236 
[52] N. Herisanu and V. Marinca, "An efficient analytical approach to investigate the dynamics of a misaligned multirotor system," Mathematics, Vol. 8, No. 7, p. 1083, Jul. 2020, https://doi.org/10.3390/math8071083

[53] S. P. Mogal and D. I. Lalwani, "Experimental investigation of unbalance and misalignment in rotor bearing system using order analysis," Journal of Measurements in Engineering, Vol. 3, No. 4, pp. 114-122, Dec. 2015.

[54] J. Lin et al., "Effects of misalignment on the nonlinear dynamics of a two-shaft rotor-bearing-gear coupling system with rub-impact fault," Journal of Vibroengineering, Vol. 19, No. 8, pp. 5960-5977, Dec. 2017, https://doi.org/10.21595/jve.2017.18476

[55] F. Curà and A. Mura, "Experimental and theoretical investigation about reaction moments in misaligned splined couplings," Mechanical Systems and Signal Processing, Vol. 45, No. 2, pp. 504-512, Apr. 2014, https://doi.org/10.1016/j.ymssp.2013.12.005

[56] N. H. Chandra and A. S. Sekhar, "Detection and monitoring of shaft misalignment in rotors using Hilbert Huang transform," in ASME Turbo Expo 2014: Turbine Technical Conference and Exposition, Jun. 2014, https://doi.org/10.1115/gt2014-25437

[57] A. Umbrajkaar and A. Krishnamoorthy, "Vibration analysis using wavelet transform and fuzzy logic for shaft misalignment," Journal of Vibroengineering, Vol. 20, No. 8, pp. 2855-2865, Dec. 2018, https://doi.org/10.21595/jve.2018.19611

[58] J. Zhou, H. Xiao, C. Li, J. Xiao, W. Zhang, and W. Fu, "Shaft orbit identification for rotating machinery based on statistical fuzzy vector chain code and support vector machine," Journal of Vibroengineering, Vol. 16, No. 2, pp. 713-724, Mar. 2014.

[59] A. K. Verma, S. Sarangi, and M. H. Kolekar, "Experimental investigation of misalignment effects on rotor shaft vibration and on stator current signature," Journal of Failure Analysis and Prevention, Vol. 14, No. 2, pp. 125-138, Apr. 2014, https://doi.org/10.1007/s11668-014-9785-7

[60] J. L. Ferrando Chacon, E. Artigao Andicoberry, V. Kappatos, G. Asfis, T.-H. Gan, and W. Balachandran, "Shaft angular misalignment detection using acoustic emission," Applied Acoustics, Vol. 85, pp. 12-22, Nov. 2014, https://doi.org/10.1016/j.apacoust.2014.03.018

[61] M. Chandra Sekhar Reddy and A. S. Sekhar, "Detection and monitoring of coupling misalignment in rotors using torque measurements," Measurement, Vol. 61, pp. 111-122, Feb. 2015, https://doi.org/10.1016/j.measurement.2014.10.031

[62] S. Fatima, A. R. Mohanty, and V. A. Naikan, "A misalignment detection methodology by measuring rate of temperature rise of shaft coupling using thermal imaging," Proceedings of the Institution of Mechanical Engineers, Part O: Journal of Risk and Reliability, Vol. 229, No. 3, pp. 209-219, Jun. 2015, https://doi.org/10.1177/1748006x15573683

[63] A. R. Mohanty and S. Fatima, "Shaft misalignment detection by thermal imaging of support bearings," IFAC-PapersOnLine, Vol. 48, No. 21, pp. 554-559, 2015, https://doi.org/10.1016/j.ifacol.2015.09.584

[64] O. Tonks and Q. Wang, "The detection of wind turbine shaft misalignment using temperature monitoring," CIRP Journal of Manufacturing Science and Technology, Vol. 17, pp. 71-79, May 2017, https://doi.org/10.1016/j.cirpj.2016.05.001

[65] M. A. Hili, T. Fakhfakh, L. Hammami, and M. Haddar, "Shaft misalignment effect on bearings dynamical behavior," The International Journal of Advanced Manufacturing Technology, Vol. 26, No. 5-6, pp. 615-622, Sep. 2005, https://doi.org/10.1007/s00170-004-2013-y

[66] M. A. Hili, T. Fakhfakh, and M. Haddar, "Failure analysis of a misaligned and unbalanced flexible rotor," Journal of Failure Analysis and Prevention, Vol. 6, No. 4, pp. 73-82, Aug. 2006, https://doi.org/10.1361/154770206x117540

[67] J. M. Bossio, G. R. Bossio, and C. H. de Angelo, "Angular misalignment in induction motors with flexible coupling," in IECON 2009 - 35th Annual Conference of IEEE Industrial Electronics (IECON 2009), pp. 1033-1038, Nov. 2009, https://doi.org/10.1109/iecon.2009.5414696 